\title{
Desempenho agronômico de genótipos de alface americana no Submédio do Vale do São Francisco
}

\author{
Jony E Yuri'; Geraldo M Resende ${ }^{1}$; Nivaldo D Costa ${ }^{1}$; Adriano S Gomes ${ }^{2}$ \\ 'Embrapa Semiárido, Petrolina-PE, Brasil; jony.yuri@embrapa.br; geraldo.milanez@embrapa.br; nivaldo.duarte@embrapa.br; \\ ${ }^{2}$ Universidade de Pernambuco (UPE), Petrolina-PE, Brasil; adrianogoms@gmail.com
}

\section{RESUMO}

Entre os tipos de alface cultivados atualmente, tem-se destacado a alface americana, que visa atender, principalmente, as redes de fast foods. Com o objetivo de avaliar o comportamento produtivo de cultivares de alface americana nas condições do Submédio do Vale do São Francisco, foram conduzidos dois experimentos, em 2013 e 2014, em Petrolina-PE. Utilizou-se o delineamento de blocos ao acaso com doze genótipos em 2013 e vinte e dois genótipos em 2014, com três repetições. Foram avaliados produção total e comercial por planta; produtividade total e comercial; circunferência da "cabeça" comercial e comprimento do caule em cada ano de cultivo. Para as cultivares avaliadas nos dois anos, efetuou-se a análise conjunta. Em 2013 a produção e produtividade total apresentaram diferenças significativas entre tratamentos, sobressaindo-se os genótipos Havassu (940 g/planta e 78,3 t/ha), Perovana (922 g/planta e 76,8 t/ha), Laurel ( $903 \mathrm{~g} /$ planta e 75,2 t/ha) e Balsamo (875 g/planta e 72,8 t/ha). Já no cultivo de 2014, a massa fresca e produtividade total variou entre 526 e $816 \mathrm{~g} /$ planta e 43,8 e 67,9 t/ha, com melhores respostas produtivas para os genótipos Laurel (816 g/planta e 67,9 t/ha) e Silvana (809 $\mathrm{g} /$ planta e 67,4 t/ha), sem contudo diferirem entre si. Pela análise conjunta, pôde-se verificar que todas as características avaliadas foram afetadas significativamente pela interação entre os fatores ano e cultivares. Conclui-se que é viável o cultivo dessa hortaliça na região. As cultivares Ironwood, Havassu, Laurel, Flora, Perovana, Raider Plus, Balsamo e Silvana são as mais indicadas.

Palavras-chave: Lactuca sativa, rendimento, competição, adaptação.

\begin{abstract}
Agronomic performance of crisphead lettuce genotypes at Sub-Middle São Francisco Valley

Among the types of lettuce currently cultivated, crisphead lettuce has exceeded, which aims to serve mainly the networks of fast food. In order to evaluate the productive behavior of lettuce cultivars in Sub-Middle of São Francisco Valley, Pernambuco State, Brazil, two experiments were carried out, in 2013 and 2014. Randomized blocks with twelve genotypes in 2013 and twenty-two genotypes in 2014, with three replications, were evaluated. Total and commercial production per plant; total and commercial productivity; circumference of the commercial "head" and stem length in each year of cultivation were obtained. On both experiments the cultivars were evaluated through joint analysis. For the 2013 crop, total production and productivity showed significant differences between treatments, wherein Havassu (940 g/plant and 78.3 t/ha), Perovana (922 g/plant and $76.8 \mathrm{t} / \mathrm{ha})$, Laurel (903 g/plant and $75.2 \mathrm{t} / \mathrm{ha}$ ) and Balsamo (875 g/ plant and $72.8 \mathrm{t} / \mathrm{ha}$ ) were the genotypes that exceeded. In 2014, fresh mass and total productivity ranged between 526 and $816 \mathrm{~g} /$ plant and 43.8 and 67.9 t/ha, Laurel (816 g/plant and 67.9 t/ha) and Silvana (809 $\mathrm{g} /$ plant and $67.4 \mathrm{t} / \mathrm{ha}$ ) presenting the best results, with no difference among them. For joint analysis, every evaluated characteristic was significantly affected by the interaction between the factors, year and cultivars. We concluded that the cultivation of crisphead lettuce is viable in the region. The cultivars Ironwood, Havassu, Laurel, Flora, Perovana, Raider Plus, Balsamo and Silvana are the most indicated.
\end{abstract}

Keywords: Lactuca sativa, yield, competition, adaptation.

(Recebido para publicação em 23 de fevereiro de 2016; aceito em 5 de outubro de 2016) (Received on February 23, 2016; accepted on October 5, 2016)

$\mathrm{O}^{\prime}$ riginária da região do mediterrâneo, a alface (Lactuca sativa) é a hortaliça folhosa mais importante no mundo e a mais comercializada no Brasil, sendo consumida, principalmente, in natura, na forma de saladas (Sala \& Costa, 2012, Santi et al., 2013) e constitui-se na espécie mais popular dentre aquelas em que as folhas são consumidas cruas e ainda frescas (Cometti et al., 2004). Entre os tipos de alface cultivados atualmente, tem-se destacado a alface americana, que visa atender, principalmente, as redes de fast foods (Yuri et al., 2002a). Tem-se constatado maior demanda dessa alface e aceitação pelo mercado consumidor brasileiro, na forma de salada (Resende et al., 2007; Sala \& Costa, 2012). Nos Estados Unidos, esse grupo de alface denominado crisphead lettuce, é considerado o mais importante dentre as hortaliças para ser consumido em forma de saladas cruas, tendo um consumo per capita superior a 11,3 kg/ano (Sanders, 2016).

De acordo com Hortbrasil (2013), a participação da alface americana no mercado brasileiro está crescendo ano após ano, passando de 26\% em 2002, para 36\% em 2012. Esse destaque se deve, principalmente, às características apresentadas por este grupo, que se diferencia dos demais por apresentar folhas externas de coloração verde-escura, folhas internas de coloração amarela ou branca, imbricadas, semelhantes ao repolho e crocantes, além de apresentar maior duração pós-colheita, possibilitando o transporte a longas distâncias 
(Yuri et al., 2002a).

É uma planta bastante influenciada por condições ambientais. A cultura é adaptada a temperaturas amenas, sendo que o ideal para seu desenvolvimento está na faixa de 15,5 a $18,3^{\circ} \mathrm{C}$, apesar de tolerar temperaturas de 26,6 a $29,4^{\circ} \mathrm{C}$, por alguns dias, desde que as temperaturas noturnas sejam baixas (Sanders, 2016). Temperaturas na faixa de 21,1 a $26,6^{\circ} \mathrm{C}$ por longos períodos promovem a elongação do caule e prejudicam a formação de "cabeças" comerciais. A temperatura ideal para o desenvolvimento da alface americana está em torno de $23^{\circ} \mathrm{C}$ durante o dia e $7^{\circ} \mathrm{C}$ à noite (Jackson et al., 2016). Temperaturas muito elevadas podem provocar queima de bordas das folhas externas, formar "cabeças" pouco compactas e também contribuir para a ocorrência de deficiência de cálcio, desordem fisiológica conhecida como tip-burn (Jackson et al., 2016).

Muitas cultivares de alface americana foram desenvolvidas e utilizadas ao longo dessas últimas décadas de plantio no Brasil. Destaque para as cultivares Great Lakes, Lorca, Raider, Raider Plus e Laurel que são caracterizadas por apresentarem "cabeça" compacta e ideais para o processamento. Laurel tem sido a cultivar mais plantada no país nas principais regiões produtoras, principalmente no período de temperaturas amenas (Sala \& Costa, 2012).

Avaliando diferentes cultivares em cultivo de inverno, Hotta (2008) verificou valores de massa fresca da "cabeça" comercial variando de 601,1 a $845,7 \mathrm{~g} /$ planta, com maior resposta para a cultivares Rafaela (845,7 g/planta), seguida pelas cultivares Laurel (796,0 g/planta), Rubette (767,4 g/planta) e Raider Plus (758,5 g/planta), sem diferenças significativas entre si. Sediyama et al. (2009) observaram, para cultivares de alface americana, em cultivo hidropônico, massa fresca total de diferentes cultivares variando de 285,6 a $445,8 \mathrm{~g} /$ planta e massa fresca comercial de 241,7 a 388,3 $\mathrm{g} /$ planta, enquanto o comprimento do caule variou de 2,9 a $4,2 \mathrm{~cm}$.

Nesse contexto, objetivou-se com o presente trabalho avaliar o comportamento de cultivares de alface americana nas condições do Submédio do Vale do
São Francisco, em dois anos de cultivo.

\section{MATERIAL E MÉTODOS}

Os experimentos foram conduzidos nos períodos de maio a julho de 2013 e abril a julho de 2014, no Campo Experimental de Bebedouro, da Embrapa Semiárido, Petrolina-PE $\left(9^{\circ} 9^{\prime} \mathrm{S}\right.$, $40^{\circ} 29^{\prime} \mathrm{O}, 365,5 \mathrm{~m}$ de altitude). Segundo a classificação climática de Köppen, a região apresenta clima do tipo BSWh', semiárido, e os seguintes valores médios anuais das variáveis climatológicas: temperatura do $a r=26,5^{\circ} \mathrm{C}$, precipitação pluvial $=541,1 \mathrm{~mm}$, umidade relativa do ar $=65,9 \%$, evaporação do tanque classe " $\mathrm{A}$ " $=2.500 \mathrm{~mm} /$ ano e velocidade do vento $=2,3 \mathrm{~m} / \mathrm{s}$. A precipitação é irregularmente distribuída no espaço e no tempo, concentrando-se nos meses de dezembro a abril; a insolação anual é superior a $3.000 \mathrm{~h}$ (Azevedo et al., 2006). Em 2013 e 2014, respectivamente, a precipitação pluviométrica acumulada foi de 10,0 e $6,5 \mathrm{~mm}$, as médias das temperaturas máxima, mínima e média foram 30,$0 ; 18,4$ e $24,1^{\circ} \mathrm{C}$ e 28,$1 ; 17,1$ e $22,2^{\circ} \mathrm{C}$, respectivamente, e a média da umidade relativa do ar foi de 58,8 e $60,4 \%$.

O delineamento experimental utilizado foi de blocos ao acaso com doze genótipos em 2013 (Balsamo, Flora, Perovana, Eagle 01, Havassu, Heatmaster, Peroba, Irene, Ironwood, Laurel, Raider Plus e Winslow) e 22 genótipos em 2014 (Angelina, Callore, Lais, Laurel, Lucy Brown, Madras RZ, Raider Plus, Silvana, Balsamo, Eagle-01, Flora, Greise, Irene, Jéssica-II, Perovana, Mayumi, 10Y1734, 10Y1734-1, 10Y1739, 11Y1921-1, 11Y1953-1 e 13B122-1), com três repetições. Salienta-se que no segundo ano, foram adicionados novos genótipos com o intuito de avaliar se os mesmos apresentariam potencial produtivo na região.

O semeio das cultivares foi realizado em 21/05/2013 e 22/04/2014, em bandejas de isopor contendo 288 células, preenchidas com substrato comercial "Plantmax HT". Ressalta-se que a diferença nas datas de semeio de um ano para o outro foi com o intuito de ajustar o cultivo em condições de temperaturas mais amenas. As mudas foram conduzidas em viveiro durante 28 dias, quando então foram transplantadas para o campo. O preparo do solo constou de aração, gradagem e levantamento dos canteiros a $20 \mathrm{~cm}$ de altura.

As parcelas experimentais constituíram-se de canteiros com quatro linhas de 2,1 m de comprimento, espaçadas de 30 $\mathrm{cm}$, sendo a distância entre plantas de 30 $\mathrm{cm}$. As duas linhas centrais, descartando duas plantas de cada extremidade das linhas, representaram a área útil das parcelas. Salienta-se, que a distância entre as bordaduras de dois canteiros vizinhos foi de $70 \mathrm{~cm}$.

$\mathrm{O}$ solo da área experimental foi classificado como Argissolo Amarelo Eutrófico de textura arenosa (Santos et al., 2006). Este foi previamente caracterizado quanto aos aspectos químicos, apresentando os seguintes resultados: $\mathrm{P}=51,43 \mathrm{mg} / \mathrm{dm}^{3} ; \mathrm{K}=1,30 \mathrm{cmol} / \mathrm{dm}^{3}$; $\mathrm{Ca}=0,70 \mathrm{cmol}_{\mathrm{c}} / \mathrm{dm}^{3} ; \mathrm{Mg}=0,40 \mathrm{cmol}_{\mathrm{c}} /$ $\mathrm{dm}^{3} ; \mathrm{H}+\mathrm{Al}=2,24 \mathrm{cmol}_{\mathrm{c}} / \mathrm{dm}^{3} ; \mathrm{CTC}$ efetiva $=4,73 \mathrm{cmol} / \mathrm{dm}^{3} ; \mathrm{V} \%=52,64$; $\mathrm{pH}$ em água $(1: 2,5)=6,78 ; \mathrm{B}=0,90 \mathrm{mg} /$ $\mathrm{dm}^{3} ; \mathrm{Cu}=0,80 \mathrm{mg} / \mathrm{dm}^{3} ; \mathrm{Fe}=14,90 \mathrm{mg} /$ $\mathrm{dm}^{3} ; \mathrm{Mn}=41,30 \mathrm{mg} / \mathrm{dm}^{3} ; \mathrm{Zn}=3,50 \mathrm{mg} /$ $\mathrm{dm}^{3}$. A adubação de plantio, com base na análise de solo, constou da aplicação de $60,3 \mathrm{~kg} / \mathrm{ha}$ de $\mathrm{N}$ e $21,3 \mathrm{~kg} / \mathrm{ha}$ de $\mathrm{P}_{2} \mathrm{O}_{5}$ no plantio. Em adubação de cobertura, foram adicionados $38,0 \mathrm{~kg} / \mathrm{ha}$ de $\mathrm{N}$ e 26,0 kg/ha de Ca (Pereira, 2015).

A cultura foi mantida no limpo por meio de capinas manuais, quando necessárias. As irrigações foram feitas utilizando-se o método de microaspersão, com turno de dois dias e lâminas de água em torno de 10-11 mm, calculadas em função da evaporação do tanque classe A, e os tratos fitossanitários foram os comumente utilizados na cultura, segundo Yuri et al. (2002a).

A colheita foi realizada em julho, aos 42 e 44 dias após o transplante, respectivamente nos anos de 2013 e 2014, quando as plantas apresentaram-se completamente desenvolvidas, sendo avaliadas a massa fresca total e comercial ( $\mathrm{g} /$ planta); produtividade total e comercial $(\mathrm{t} / \mathrm{ha})$, circunferência da "cabeça" comercial e comprimento do caule $(\mathrm{cm})$. Como massa fresca e produtividade total considerou-se a planta inteira e como comercial, a "cabeça", 
retirando-se as folhas da base da planta. Os dados coletados foram submetidos à análise de variância, utilizando-se o teste $\mathrm{F}$ para comparação dos quadrados médios a $5 \%$ de probabilidade e as médias foram comparadas pelo teste de Scott-Knott, a 5\% de probabilidade, empregando-se o programa SISVAR 5.0 (Ferreira, 2010). Posteriormente, para as cultivares testadas nos dois anos, efetuou-se a análise conjunta.

\section{RESULTADOS E DISCUSSÃO}

A massa fresca total por planta e a produtividade total mostraram diferenças significativas entre cultivares, sobressaindo-se os genótipos Havassu (940 g/planta e 78,3 t/ha), Perovana (922 g/planta e 76,8 t/ha), Laurel (903 $\mathrm{g} /$ planta e 75,2 t/ha) e Balsamo (875 g/ planta e 72,8 t/ha) como os melhores desempenhos, sem, contudo, diferirem entre si, em 2013 (Tabela 1). Para o cultivo de 2014, a massa fresca total e a produtividade total variaram entre 526 e $816 \mathrm{~g} / \mathrm{planta}$ e 43,8 e $67,9 \mathrm{t} / \mathrm{ha}$, com melhores respostas produtivas para os genótipos Laurel (816 g/planta e 67,9 t/ ha) e Silvana ( $809 \mathrm{~g} /$ planta e 67,4 t/ha), sem diferirem entre si (Tabela 2). Yuri et al. (2004), em condições de inverno, observaram variações, entre 0,77 e $1,27 \mathrm{~kg} /$ planta, avaliando uma série de genótipos de alface americana, enquanto Sediyama et al. (2009) verificaram massa fresca total de diferentes cultivares inferiores, variando entre 285,6 a 445,8 $\mathrm{g} /$ planta. Salienta-se que, nesses ensaios, os genótipos não foram os mesmos avaliados no presente trabalho, em função da dinâmica no processo de seleção de novas cultivares.

Os resultados obtidos pelos genótipos mais produtivos podem ser justificados pela ação do genótipo e do ambiente, pois as plantas respondem de maneira distinta aos fatores ambientais e às práticas agrícolas (Silva et al., 2000). Salientado ainda por Echer et al. (2001), que a escolha criteriosa da cultivar é decisiva para o sucesso do sistema de cultivo adotado. Outros fatores relevantes são as condições ambientais. Nesse quesito, as temperaturas médias obtidas durante a execução dos experimentos a campo em 2013 e 2014 foram 24,1 e $22,2^{\circ} \mathrm{C}$, com mínimas de 18,4 e $17,1^{\circ} \mathrm{C}$ e máximas de 30,0 e $28,1^{\circ} \mathrm{C}$, umidade relativa de 58,8 e $60,4 \%$ e baixa precipitação no período de 10,0 e 6,5 mm. Apesar de não satisfazerem plenamente as condições ideais preconizadas por Sanders (2016), não se mostraram limitantes ao cultivo dessa hortaliça, o que pode ser comprovado pelas boas produtividades alcançadas.

Os genótipos comportaram-se diferentemente quanto à massa fresca comercial por planta e a produtividade comercial em 2013 (Tabela 1). Os genótipos Ironwood (587 g/planta e 48,9 t/ha), Perovana (560 g/planta e 46,7 t/ha), Havassu (535 g/planta e 44,6 t/ha), Laurel (524 g/planta e 43,7 t/ha), Balsamo (512 g/planta e 42,7 t/ ha), Flora (506 g/planta e 42,2 t/ha), Winslow (497 g/planta e 41,4 t/ha) e Raider Plus (494 g/planta e 41,2 t/ha) foram os mais produtivos, não diferenciando entre si. Em 2014, os genótipos Laurel, com $500 \mathrm{~g} /$ planta e 41,6 t/ha e 10Y1921-1, com 488 g/planta e 40,6 t/ ha, apresentaram os maiores valores, salientando-se que houve oscilações entre 267 e $500 \mathrm{~g} /$ planta e 22,2 e 41,6 $\mathrm{t} / \mathrm{ha}$, respectivamente, entre genótipos para essas características. Hotta (2008) verificou valores de massa fresca da "cabeça" comercial variando de 601,1 a $845,7 \mathrm{~g}$, com maiores respostas desta característica para as cultivares Rafaela (845,7 g/planta), seguidas pelas cultivares Laurel (796,0 g/planta), Rubette (767,4 g/planta) e Raider Plus (758,5 $\mathrm{g} /$ planta). Yuri et al. (2004) verificaram para as cultivares mais responsivas em massa fresca comercial, oscilações entre 565 e $729 \mathrm{~g} /$ planta.

Tabela 1. Produção e produtividade total e comercial, comprimento do caule e circunferência da "cabeça" comercial de cultivares de alface americana (total and marketable production and yield, stem length and commercial head circumference of crisphead lettuce cultivars). Petrolina, Embrapa Semiárido, 2013.

\begin{tabular}{|c|c|c|c|c|c|c|}
\hline \multirow{2}{*}{ Cultivares } & \multicolumn{2}{|c|}{ Produção (g/planta) } & \multicolumn{2}{|c|}{ Produtividade (t/ha) } & \multirow{2}{*}{$\begin{array}{l}\text { Comprimento } \\
\text { do caule }(\mathrm{cm})\end{array}$} & \multirow{2}{*}{$\begin{array}{c}\text { Circunferência } \\
\text { da "cabeça" (cm) }\end{array}$} \\
\hline & Total & Comercial & Total & Comercial & & \\
\hline Balsamo & $875 \mathrm{a}$ & $512 \mathrm{a}$ & $72,8 \mathrm{a}$ & $42,7 \mathrm{a}$ & $10,2 \mathrm{a}$ & $45,6 \mathrm{a}$ \\
\hline Flora & $822 \mathrm{~b}$ & $506 \mathrm{a}$ & $68,5 \mathrm{~b}$ & $42,2 \mathrm{a}$ & $9,1 \mathrm{~b}$ & $46,0 \mathrm{a}$ \\
\hline Perovana & $922 \mathrm{a}$ & $560 \mathrm{a}$ & $76,8 \mathrm{a}$ & $46,7 \mathrm{a}$ & $8,3 \mathrm{~b}$ & $46,2 \mathrm{a}$ \\
\hline Eagle 01 & $821 \mathrm{~b}$ & $439 \mathrm{~b}$ & $68,4 \mathrm{~b}$ & $36,6 \mathrm{~b}$ & $10,0 \mathrm{a}$ & $45,9 \mathrm{a}$ \\
\hline Havassu & $940 \mathrm{a}$ & $535 \mathrm{a}$ & $78,3 \mathrm{a}$ & $44,6 \mathrm{a}$ & $8,3 \mathrm{~b}$ & $46,3 \mathrm{a}$ \\
\hline Heatmaster & $801 \mathrm{~b}$ & $457 \mathrm{~b}$ & $66,7 \mathrm{~b}$ & $38,1 \mathrm{~b}$ & $7,5 \mathrm{~b}$ & $44,9 \mathrm{~b}$ \\
\hline Peroba & $811 \mathrm{~b}$ & $455 \mathrm{~b}$ & $67,6 \mathrm{~b}$ & $37,9 \mathrm{~b}$ & $10,4 \mathrm{a}$ & $44,9 \mathrm{~b}$ \\
\hline Irene & $807 \mathrm{~b}$ & $425 \mathrm{~b}$ & $67,2 \mathrm{~b}$ & $35,4 \mathrm{~b}$ & $8,0 \mathrm{~b}$ & $46,2 \mathrm{a}$ \\
\hline Ironwood & $847 \mathrm{~b}$ & $587 \mathrm{a}$ & $70,6 \mathrm{~b}$ & $48,9 \mathrm{a}$ & $7,3 \mathrm{~b}$ & $45,8 \mathrm{a}$ \\
\hline Laurel & $903 \mathrm{a}$ & $524 \mathrm{a}$ & $75,2 \mathrm{a}$ & $43,7 \mathrm{a}$ & $9,0 \mathrm{~b}$ & $44,5 \mathrm{~b}$ \\
\hline Raider Plus & $796 \mathrm{~b}$ & $494 \mathrm{a}$ & $63,3 \mathrm{~b}$ & $41,2 \mathrm{a}$ & $8,4 \mathrm{~b}$ & $44,2 \mathrm{~b}$ \\
\hline Winslow & $785 \mathrm{~b}$ & $497 \mathrm{a}$ & $65,4 \mathrm{~b}$ & $41,4 \mathrm{a}$ & $9,7 \mathrm{a}$ & $45,2 \mathrm{a}$ \\
\hline CV (\%) & 10,6 & 12,8 & 10,6 & 12,8 & 9,4 & 5,0 \\
\hline
\end{tabular}

Médias seguidas da mesma letra na coluna não diferem entre si pelo teste de Scott-Knott, a 5\% de probabilidade (means followed by the same letter in the column do not differ by Scott-Knott, 5\%). 
Tabela 2. Produção e produtividade total e comercial, comprimento do caule e circunferência da "cabeça" comercial de cultivares de alface americana (total and marketable production and yield, stem length and commercial head circumference of crisphead lettuce cultivars). Petrolina, Embrapa Semiárido, 2014.

\begin{tabular}{|c|c|c|c|c|c|c|}
\hline \multirow{2}{*}{ Cultivares } & \multicolumn{2}{|c|}{ Produção (g/planta) } & \multicolumn{2}{|c|}{ Produtividade (t/ha) } & \multirow{2}{*}{$\begin{array}{l}\text { Comprimento } \\
\text { do caule }(\mathrm{cm})\end{array}$} & \multirow{2}{*}{$\begin{array}{r}\text { Circunferência } \\
\text { da "cabeça" }(\mathrm{cm})\end{array}$} \\
\hline & Total & Comercial & Total & Comercial & & \\
\hline Laurel & $816 \mathrm{a}$ & $500 \mathrm{a}$ & $67,9 \mathrm{a}$ & $41,6 \mathrm{a}$ & $9,0 \mathrm{~d}$ & $44,2 \mathrm{a}$ \\
\hline Silvana & 809 a & $457 \mathrm{~b}$ & $67,4 \mathrm{a}$ & $38,1 \mathrm{~b}$ & $15,7 \mathrm{a}$ & $44,0 \mathrm{a}$ \\
\hline 10Y1921-1 & $741 \mathrm{~b}$ & $488 \mathrm{a}$ & $61,7 \mathrm{~b}$ & $40,6 \mathrm{a}$ & $15,0 \mathrm{~b}$ & $39,7 \mathrm{c}$ \\
\hline Flora & $705 \mathrm{~b}$ & $405 \mathrm{c}$ & $58,7 \mathrm{~b}$ & $33,7 \mathrm{c}$ & $8,3 \mathrm{~d}$ & $43,0 \mathrm{a}$ \\
\hline Raider Plus & $688 \mathrm{~b}$ & $457 \mathrm{~b}$ & $57,2 \mathrm{~b}$ & $38,0 \mathrm{~b}$ & $11,5 \mathrm{c}$ & $40,7 \mathrm{~b}$ \\
\hline Balsamo & $687 \mathrm{~b}$ & $418 \mathrm{c}$ & $57,2 \mathrm{~b}$ & $34,8 \mathrm{c}$ & $10,0 \mathrm{c}$ & $44,0 \mathrm{a}$ \\
\hline Eagle-01 & $680 \mathrm{~b}$ & $354 \mathrm{~d}$ & $56,6 \mathrm{~b}$ & $29,5 \mathrm{~d}$ & $11,0 \mathrm{c}$ & $42,8 \mathrm{a}$ \\
\hline Mayumi & $680 \mathrm{~b}$ & $460 \mathrm{~b}$ & $56,6 \mathrm{~b}$ & $38,3 \mathrm{~b}$ & $9,3 \mathrm{~d}$ & $43,3 \mathrm{a}$ \\
\hline 13B122-1 & $650 \mathrm{c}$ & $375 d$ & $54,1 \mathrm{c}$ & $31,2 \mathrm{~d}$ & $10,3 \mathrm{c}$ & $41,7 \mathrm{~b}$ \\
\hline Madras RZ & $648 \mathrm{c}$ & $370 \mathrm{~d}$ & $54,0 \mathrm{c}$ & $30,8 \mathrm{~d}$ & $14,3 \mathrm{~b}$ & $42,0 \mathrm{~b}$ \\
\hline Greise & $637 c$ & $429 c$ & $53,0 \mathrm{c}$ & $35,7 \mathrm{c}$ & $13,7 \mathrm{~b}$ & $43,7 \mathrm{a}$ \\
\hline 11Y1953-1 & $625 \mathrm{c}$ & $342 \mathrm{~d}$ & $52,0 \mathrm{c}$ & $28,4 \mathrm{~d}$ & $11,0 \mathrm{c}$ & $39,7 \mathrm{c}$ \\
\hline 10Y1734-1 & $621 \mathrm{c}$ & $370 \mathrm{~d}$ & $50,0 \mathrm{c}$ & $30,8 \mathrm{~d}$ & $8,0 \mathrm{e}$ & $39,7 \mathrm{c}$ \\
\hline Perovana & $617 c$ & $369 d$ & $51,3 \mathrm{c}$ & $30,7 \mathrm{~d}$ & $6,2 \mathrm{f}$ & $40,0 \mathrm{~b}$ \\
\hline Jessica-II & $597 \mathrm{c}$ & $377 \mathrm{~d}$ & $49,7 \mathrm{c}$ & $31,4 \mathrm{~d}$ & $16,3 \mathrm{a}$ & $42,0 \mathrm{~b}$ \\
\hline Callore & $572 \mathrm{~d}$ & $370 \mathrm{~d}$ & $47,6 \mathrm{~d}$ & $30,8 \mathrm{~d}$ & $9,0 \mathrm{~d}$ & $41,3 \mathrm{~b}$ \\
\hline Lucy Brown & $569 \mathrm{~d}$ & $269 \mathrm{e}$ & $47,4 \mathrm{~d}$ & $22,4 \mathrm{e}$ & $7,7 \mathrm{e}$ & $42,7 \mathrm{a}$ \\
\hline Irene & $549 \mathrm{~d}$ & $295 \mathrm{e}$ & $45,7 \mathrm{~d}$ & $24,5 \mathrm{e}$ & $9,0 \mathrm{~d}$ & $41,3 \mathrm{~b}$ \\
\hline 10Y1734 & $548 \mathrm{~d}$ & $278 \mathrm{e}$ & $45,6 \mathrm{~d}$ & $23,1 \mathrm{e}$ & $5,0 \mathrm{f}$ & $44,0 \mathrm{a}$ \\
\hline $10 Y 1739$ & $545 \mathrm{~d}$ & $286 \mathrm{e}$ & $45,3 \mathrm{~d}$ & $23,8 \mathrm{e}$ & $6,0 \mathrm{f}$ & $41,7 \mathrm{~b}$ \\
\hline Lais & $533 \mathrm{~d}$ & $326 \mathrm{~d}$ & $44,3 \mathrm{~d}$ & $27,2 \mathrm{~d}$ & $8,7 \mathrm{~d}$ & $39,3 \mathrm{~d}$ \\
\hline Angelina & $526 \mathrm{~d}$ & $267 \mathrm{e}$ & $43,8 \mathrm{~d}$ & $22,2 \mathrm{e}$ & $5,3 \mathrm{f}$ & $39,3 \mathrm{~d}$ \\
\hline $\mathrm{CV}(\%)$ & 10,6 & 9,7 & 9,5 & 9,7 & 12,6 & 5,9 \\
\hline
\end{tabular}

Médias seguidas da mesma letra na coluna não diferem entre si pelo teste de Scott-Knott, a 5\% de probabilidade (means followed by the same letter in the column do not differ by Scott-Knott, 5\%).

A demanda do mercado brasileiro é por dois tipos de alface americana: um tipo com "cabeça" compacta para processamento mínimo, em que a alface é lavada, picada, higienizada e embalada (indústria), direcionada para grandes redes de supermercados ou redes de lanchonetes, e o outro com formação de "cabeça" menos compacta para a venda em mercados e feiras livres. Menores comprimentos de caule são desejáveis quando o destino é a indústria de beneficiamento, devendo o mesmo ser reduzido, proporcionando menores perdas durante o processamento. O caule excessivamente comprido também acarreta uma menor compacidade da "cabeça" e dificulta o beneficiamento, afetando a qualidade final do produto (Yuri et al., 2002b; Resende et al., 2003). Caules com comprimento de até $6,0 \mathrm{~cm}$ seriam os mais adequados, sendo aceitáveis até o patamar de $9,0 \mathrm{~cm} \mathrm{e}$ inaceitáveis ou menos recomendados para processamento acima disto (Yuri et al., 2004; Resende et al., 2005). Entre os genótipos mais produtivos em 2013, destacaram-se 'Ironwood' (7,3 cm), 'Havassu', (8,3 cm), 'Perovana' (8,3 $\mathrm{cm})$, 'Raider Plus' $(8,4 \mathrm{~cm})$, 'Laurel' $(9,0 \mathrm{~cm})$ e 'Flora' $(9,1 \mathrm{~cm})$ (Tabela 1$)$. Para o cultivo em 2014, os melhores resultados quanto ao comprimento de caule foram obtidos nos genótipos pouco produtivos. Entre os que alcançaram maiores rendimentos em massa fresca e produtividade comercial, o genótipo Laurel com 9,0 cm e Mayumi com 9,3 $\mathrm{cm}$ podem ser mencionados como os que se adequaram a estes requisitos.

Para a venda em mercados e feiras livres, os critérios adotados entre os produtores de alface americana, não implicam em formar uma "cabeça" compacta (comercializada por unidade com todas as folhas), bastando apresentar formação de "cabeça" para que o produtor realize a colheita, mesmo não tendo atingido o ponto final de maturidade (Fabri \& Sala, 2007). Neste caso, os genótipos Havassu, Perovana, Laurel e Balsamo são todos recomendáveis para cultivo, em função de seus rendimentos em massa fresca e produtividade total em 2013 (Tabela 1). No cultivo em 2014 destacaram-se os genótipos Laurel e Silvana como os mais produtivos, seguidos pelos genótipos 10Y1921-1, Flora, Raider Plus, Balsamo, Eagle-01 e Mayumi, com produtividade acima de $50,0 \mathrm{t} / \mathrm{ha}$.

A circunferência da "cabeça" da alface americana é muito importante para o produtor, pois a remuneração depende do seu tamanho, pois afeta o rendimento no beneficiamento. "Cabeças" muito 
Tabela 3. Produção e produtividade total e comercial, comprimento do caule e circunferência da "cabeça" comercial de cultivares de alface americana em função do ano de produção (total and marketable production and yield, stem length and commercial head circumference of crisphead lettuce cultivars depending on year). Petrolina, Embrapa Semiárido, 2014.

\begin{tabular}{|c|c|c|c|c|c|c|}
\hline \multirow{3}{*}{ Cultivares } & \multicolumn{6}{|c|}{2013} \\
\hline & \multicolumn{2}{|c|}{ Produção (g/planta) } & \multicolumn{2}{|c|}{ Produtividade (t/ha) } & \multirow{2}{*}{$\begin{array}{l}\text { Comprimento } \\
\text { do caule }(\mathrm{cm})\end{array}$} & \multirow{2}{*}{$\begin{array}{l}\text { Circunferência da } \\
\text { "cabeça" (cm) }\end{array}$} \\
\hline & Total & Comercial & Total & Comercial & & \\
\hline Perovana & $922 \mathrm{aA}$ & $560 \mathrm{aA}$ & $76,8 \mathrm{aA}$ & $46,7 \mathrm{aA}$ & $8,3 \mathrm{aA}$ & $46,2 \mathrm{aA}$ \\
\hline Laurel & $903 \mathrm{aA}$ & $524 \mathrm{aA}$ & $75,2 \mathrm{aA}$ & $43,7 \mathrm{aA}$ & $8,8 \mathrm{aA}$ & $44,5 \mathrm{aA}$ \\
\hline Balsamo & $875 \mathrm{aA}$ & $512 \mathrm{aA}$ & $72,8 \mathrm{aA}$ & $42,7 \mathrm{aA}$ & $10,2 \mathrm{aA}$ & $45,6 \mathrm{aA}$ \\
\hline Flora & $821 \mathrm{bA}$ & $506 \mathrm{aA}$ & $68,5 \mathrm{bA}$ & $42,2 \mathrm{aA}$ & $9,1 \mathrm{aA}$ & $46,0 \mathrm{aA}$ \\
\hline Eagle-01 & $821 \mathrm{bA}$ & $439 \mathrm{bA}$ & $68,4 \mathrm{bA}$ & $36,6 \mathrm{bA}$ & $10,0 \mathrm{aA}$ & 45,9 aA \\
\hline Irene & $807 \mathrm{bA}$ & $425 \mathrm{bA}$ & $67,2 \mathrm{bA}$ & $35,4 \mathrm{bA}$ & $8,0 \mathrm{aA}$ & $46,2 \mathrm{aA}$ \\
\hline \multirow{2}{*}{ Raider Plus } & $796 \mathrm{bA}$ & $494 \mathrm{aA}$ & $66,3 \mathrm{bA}$ & $41,2 \mathrm{aA}$ & $8,4 \mathrm{aB}$ & $44,2 \mathrm{aA}$ \\
\hline & & & & 2014 & & \\
\hline Perovana & $617 \mathrm{cB}$ & $369 \mathrm{cB}$ & $51,3 \mathrm{cB}$ & $30,7 \mathrm{cB}$ & $6,2 \mathrm{cB}$ & $40,0 \mathrm{bB}$ \\
\hline Laurel & $816 \mathrm{aA}$ & $500 \mathrm{aA}$ & $67,9 \mathrm{aA}$ & $41,6 \mathrm{aA}$ & $9,0 \mathrm{bA}$ & $44,2 \mathrm{aA}$ \\
\hline Balsamo & $687 \mathrm{bB}$ & $418 \mathrm{bB}$ & $57,2 \mathrm{bB}$ & $34,8 \mathrm{bB}$ & $10,0 \mathrm{bA}$ & $44,0 \mathrm{aA}$ \\
\hline Flora & $705 \mathrm{bB}$ & $405 \mathrm{bB}$ & $57,8 \mathrm{bB}$ & $33,7 \mathrm{bB}$ & $8,3 \mathrm{bA}$ & $43,0 \mathrm{aB}$ \\
\hline Eagle-01 & $680 \mathrm{bB}$ & $354 \mathrm{cB}$ & $56,6 \mathrm{bB}$ & $29,5 \mathrm{cB}$ & $11,0 \mathrm{aA}$ & $42,8 \mathrm{aB}$ \\
\hline Irene & $549 \mathrm{cB}$ & $295 \mathrm{~dB}$ & $45,7 \mathrm{cB}$ & $24,5 \mathrm{~dB}$ & $9,0 \mathrm{bA}$ & $41,3 \mathrm{bB}$ \\
\hline Raider Plus & $688 \mathrm{bB}$ & $457 \mathrm{aA}$ & $57,2 \mathrm{bB}$ & $38,0 \mathrm{aA}$ & $11,5 \mathrm{aA}$ & $40,7 \mathrm{bB}$ \\
\hline CV (\%) (2013 e 2014) & 7,08 & 6,83 & 7,09 & 6,83 & 12,26 & 2,36 \\
\hline
\end{tabular}

Médias seguidas de mesma letra minúscula em cada coluna e letra maiúscula em cada linha, não diferem entre si pelo teste de Scott-Knott, a $5 \%$ de probabilidade (means followed by the same lowercase letter in each collumn and uppercase letter in each line, do not differ by Scott-Knott, 5\%).

pequenas diminuem o rendimento dos operadores e aumentam o material de descarte durante o processamento (Yuri, 2000). Nesse contexto, todas as cultivares apresentaram boa circunferência, com pequena variação entre 44,2 e 46,3 $\mathrm{cm}$ em 2013 (Tabela 1), assim como em 2014 quando as maiores circunferências se situaram entre 43,0 e $44,2 \mathrm{~cm}$ (Tabela 2).

Com relação às sete cultivares que foram avaliadas em ambos os anos (Balsamo, Flora, Perovana, Eagle-01, Irene, Laurel e Raider Plus), pôde-se verificar que todas as características avaliadas foram afetadas significativamente pela interação entre os fatores ano e cultivares (Tabela 3). Em termos de produção total por planta e produtividade total, observou-se que apenas a cultivar Laurel teve comportamento similar nos dois anos, enquanto que as demais foram superiores no ano de 2013. Comportamento semelhante foi verificado também nas características produção comercial por planta e produtividade comercial; entretanto, além da cv. Laurel, a Raider também se mostrou estável nessas características.

Constatou-se que a maioria das cultivares não apresentou diferenças significativas no comprimento de caule entre os anos de cultivo. Constituíram exceção as cultivares Raider Plus, com maior comprimento no ano de 2014, e a Perovana, com resultado oposto, ou seja, maior caule no cultivo de 2013. $\mathrm{O}$ fato de a temperatura média ter sido menor no ano de 2014, pode sugerir que a cv. Perovana seja um genótipo mais tardio, enquanto que a cv. Raider Plus apresentou comportamento oposto, ou seja, maior precocidade. Sugere-se que a 'Perovana', que teve o menor crescimento de caule, deveria ser colhida mais tarde, visando maior produtividade. Quanto à circunferência de "cabeça", com exceção das cultivares Laurel e Balsamo, que não apresentaram diferenças significativas, as demais apresentaram maior valor em 2013.

Em função dos resultados obtidos nos dois anos de cultivo, pode-se concluir que é viável o cultivo dessa hortaliça nas condições do Submédio do Vale do São Francisco. Os genótipos Ironwood, Havassu, Laurel, Flora, Perovana, Raider Plus, Balsamo e Silvana são os mais indicados. Salienta-se ainda que, pela sua boa produtividade (acima de 50,0 t/ha), os genótipos 10Y1921-1, Eagle-01 e Mayumi são também opções potenciais de cultivo de alface americana para as condições região.

\section{REFERÊNCIAS}

AZEVEDO, PV; SOUSA, IF; SILVA, BB; SILVA, VPR. 2006. Water-use efficiency of dwarfgreen coconut (Cocos nucifera L.) orchards in Northeast Brazil. Agricultural Water Management 1: 259-264.

COMETTI, NN; MATIAS, GCS; ZONTA, E; MARY, W; FERNANDES, MS. 2004. Compostos nitrogenados e açúcares solúveis em tecidos de alface orgânica, hidropônica e convencional. Horticultura Brasileira 22: 748-753.

ECHER, MM; SIGRIST, JMM; GUIMARÃES, VF; MINAMI, K. 2001. Comportamento de cultivares de alface em função do espaçamento. Revista de Agricultura 76: 267-275.

FABRI, EG; SALA, FC. 2007. Alface americana: 
Aponta mercado crescente. Revista Campo \& Negócios HF 27: 27-28.

FERREIRA, DF. 2010. SISVAR Versão 5.3. Lavras: Departamento de Ciências Exatas, UFLA.

HORTBRASIL. 2013. Alface na CEAGESP paulistana. Disponível em: $<$ http://www. hortibrasil.org.br/jnw/index.php?option=com content\&view $=$ article \&id=1132:alface-naceagesp-paulistana-i\&catid $=50$ : comercia lizacao\&Itemid $=82>$. Acessado em 15 de fevereiro 2016.

HOTTA, LFK. 2008. Interação de progênies de alface do grupo americano por épocas e cultivo. Botucatu: UNESP. 87p. (Dissertação mestrado).

JACKSON, L; MAYBERRY, K; LAEMMLEN, F; KOIKE, S; SCHLUBACK, K. Iceberg lettuce production in California. Disponível em: <anrcatalog.ucdavis.edu/pdf/7215.pdf>. Acessado em 15 de fevereiro 2016.

PEREIRA, AKS. 2015. Épocas de aplicação e doses de nitrato de cálcio em alface americana. Ipameri: UEGoiás. 33p (Dissertação mestrado).

RESENDE, GM; YURI, JE; CARVALHO, JG; SOUZA, RJ; RODRIGUES JUNIOR, JC; MOTA, JH. 2005. Resposta da alface americana (Lactuca sativa L.) a doses e épocas de aplicação de cobre. Ciência Agrotecnologia
29: 1209-1214.

RESENDE, GM; YURI, JE; MOTA, JH; SOUZA, RJ; FREITAS, SAC; RODRIGUES JÚNIOR, JC. 2003. Efeitos de tipos de bandejas e idade de transplantio de mudas sobre o desenvolvimento e produtividade de alface americana. Horticultura Brasileira 21: 562567.

RESENDE, GM; YURI, JE; SOUZA, RJ. 2007. Épocas de plantio e doses de silício no rendimento de alface tipo americana. Horticultura Brasileira 25: 455-459.

SALA, FC; COSTA, CP. 2012. Retrospectiva e tendência da alfacicultura brasileira. Horticultura Brasileira 30: 187-194.

SANDERS, DC. Lettuce production. Disponível em <http://www.ces.ncsu.edu/hil/hil-11. html $>$. Acessado em 15 de fevereiro 2016.

SANTI,A; SCARAMUZZA, WLMP; NEUHAUS, A; DALLACORT, R; KRAUSE, W; TIEPPO, RC. 2013. Desempenho agronômico de alface americana fertilizada com torta de filtro em ambiente protegido. Horticultura Brasileira 31: 338-343.

SANTOS, HG; JACOMINE, PKT; ANJOS, LHC; OLIVEIRA, VA; OLIVEIRA, JB; COELHO, MR; LUMBRERAS, JF; CUNHA, TJF (eds). 2006. Sistema brasileiro de classificação de solos. 2. ed. Rio de Janeiro: Embrapa Solos. $306 \mathrm{p}$.
SEDIYAMA, MAN; PEDROSA, MW; SALGADO, LT; PEREIRA, PC. 2009. Desempenho de cultivares de alface para cultivo hidropônico no verão e no inverno. Cientifica 37: 98-106.

SILVA, VF; BEZERRA NETO, F; NEGREIROS, MZ; PEDROSA, JF. 2000. Comportamento de cultivares de alface em diferentes espaçamentos sob temperatura e luminosidade elevadas. Horticultura Brasileira 18: 183-187.

YURI, JE. 2000. Avaliação de cultivares de alface americana em duas épocas de plantio em dois locais do sul de Minas Gerais. Lavras: UFLA. 51p. (Dissertação mestrado).

YURI, JE; MOTA, JH; SOUZA, RJ; RESENDE, GM; FREITAS, SAC; RODRIGUES JUNIOR, JC. 2002a. Alface americana: cultivo comercial. Lavras: UFLA. 51p. (Textos acadêmicos, 13).

YURI, JE; RESENDE, GM; MOTA, JH; SOUZA, RJ; RODRIGUES JÚNIOR, JC. 2004. Comportamento de cultivares e linhagens de alface americana em Santana da Vargem (MG), nas condições de inverno. Horticultura Brasileira 22: 322-325.

YURI, JE; SOUZA, RJ; FREITAS, SAC; RODRIGUES JÚNIOR, JC; MOTA, JH. 2002b. Comportamento de cultivares de alface tipo americana em Boa Esperança. Horticultura Brasileira 20: 229-232. 